\title{
Emergency Stop Button Device
}

National Cancer Institute

\section{Source}

National Cancer Institute. Emergency Stop Button Device. NCI Thesaurus. Code C50308.

A button and circuits designed to force the shutdown of a machine or device. 